\title{
Accuracy of insulin resistance indices for metabolic syndrome: a cross-sectional study in adults
}

\author{
Luciana Pavan Antoniolli ${ }^{*}$ [D, Bárbara Limberger Nedel ${ }^{1}$, Tassia Cividanes Pazinato', \\ Leonardo de Andrade Mesquita ${ }^{1}$ and Fernando Gerchman ${ }^{2}$
}

\begin{abstract}
Background: This study aimed to determine the ability of commonly used insulin resistance indices to identify the metabolic syndrome.

Methods: 183 people referred for outpatient care at the Metabolism Unit of Hospital de Clínicas de Porto Alegre were evaluated with anthropometric, blood pressure, lipid profile, and adiponectin measurements. Glucose tolerance status was determined by $2-\mathrm{h} \mathrm{75-g}$ oral glucose tolerance test and glycosylated hemoglobin. Definition of metabolic syndrome was based on the Joint Interim Statement of different medical associations. Twenty-one indices of insulin resistance were estimated from published equations. The accuracy of these indices was determined by area under the ROC curve (AUC) analysis. In addition, we determined an optimal cut point for each index and its performance as a diagnostic test.

Results: The study population was comprised of 183 people (73.2\% women; $78.7 \%$ white; age $52.6 \pm 12.0$ years, mean \pm standard deviation), of whom 140 (76.5\%) had metabolic syndrome. The reciprocal of the Gutt index provided the greatest AUC for identification of metabolic syndrome, but there were no statistical differences between Gutt and 11 AUC indices. Gutt presented $86.4 \%$ sensitivity and $76.7 \%$ specificity to identify metabolic syndrome.

Conclusions: A number of commonly employed indices of insulin resistance are capable of identifying individuals with the metabolic syndrome.
\end{abstract}

Keywords: Metabolic syndrome, Insulin resistance, Insulin sensitivity

\section{Background}

Insulin resistance is a condition in which a greater than normal amount of insulin is required to obtain a quantitatively normal metabolic response. Most commonly, insulin resistance is used to refer to the inability of insulin to stimulate glucose disposal [1]. Insulin resistance is also associated with the development of the metabolic syndrome [2], which represents a cluster of cardiometabolic risk factors which promote the development of cardiovascular disease and type 2 diabetes [3, 4]. The individual

\footnotetext{
*Correspondence: lucianapantoniolli@gmail.com

${ }^{1}$ Scientific Initiation Program, Faculty of Medicine, Federal University of Rio Grande do Sul, Rua Ramiro Barcelos, 2400, Second floor, Porto Alegre, RS 90035-003, Brazil

Full list of author information is available at the end of the article
}

components of the metabolic syndrome include hyperglycemia, elevated blood pressure, elevated triglyceride levels, low HDL cholesterol levels, and central obesity [5]. A recent systematic review estimated the general prevalence of metabolic syndrome of adults in Brazil in 29.6\% (range 14.9-65.3\%) [6].

Although insulin resistance has been shown to be closely associated with the metabolic syndrome and to play an important physiopathological role in the development of the individual components of the metabolic syndrome [3], quantification of insulin resistance in general clinical practice is difficult. The most precise method for quantitating insulin resistance is the hyperinsulinemic euglycemic clamp technique, because it directly measures the effects of insulin to stimulate glucose utilization 
under steady-state conditions in vivo [7]. However, it involves intravenous infusion of insulin, frequent blood sampling over a 2-h period, and continuous adjustment of a glucose infusion, making it an impractical tool for large-scale epidemiological studies and clinical practice [8].

Consequently, a number of surrogate indices have been developed to estimate insulin sensitivity [8]. Both static indices, using fasting blood samples, and dynamic indices, requiring fasting and 2-h blood samples from an oral glucose tolerance test, have been developed $[9,10]$. Measurements of adiponectin and inflammatory markers have also been used as a surrogate measurement of insulin sensitivity [8]. The performance of these equations has been evaluated in different populations against a variety of measures of insulin sensitivity (Additional file 1).

Insulin resistance is a key component of the metabolic syndrome, but it is not included as part of the definition because it is not easily quantitated in clinical practice. To investigate the relationship between insulin resistance and metabolic syndrome, we evaluated the correlation between multiple surrogate indices of insulin resistance and the presence of metabolic syndrome in adult participants, aged $24-83$ years, with different degrees of glucose tolerance.

\section{Methods}

\section{Subjects}

The patient population initially consisted of 223 consecutive participants who did not have a previous diagnosis of metabolic syndrome and who were referred for outpatient care in the Metabolism Unit of Hospital de Clínicas de Porto Alegre. Forty participants were not included based on following exclusion criteria: insulin treatment, clinically significant autoimmune disease, uncompensated hypo- or hyperthyroidism, malignant disease that could affect 5-year survival, stage IV-V chronic kidney disease, AIDS, pregnancy/lactation, dementia, cirrhosis, hepatitis, glucocorticoid or anti-retroviral treatment, menopause hormone replacement therapy, and malnutrition. The remaining 183 participants were included in the analysis. Nine were on treatment with oral hypoglycemic agents. The research related to human use has been complied with all the relevant national regulations, institutional policies and in accordance to the tenets of the Helsinki Declaration, and has been approved by the authors' institutional review board. The participants provided written informed consent prior to participation.

\section{Methods}

Participants underwent a standard evaluation, which included medical history, physical examination, and anthropometric measurements. Ethnicity was based on self-reported skin color and recorded as white or nonwhite, which included black, brown, yellow, Indigenous, and undeclared, according to the national definition used in Brazil [11]. Physical activity was classified in four categories, based upon the classification proposed by Tuomilehto et al. [12]: sedentary, light exercise, moderate exercise, and heavy exercise. Waist circumference was taken at the midpoint between the lower costal margin and the iliac crest measured to the nearest $0.5 \mathrm{~cm}$.

Blood pressure was measured in the seated position 1 week after withdrawal of all antihypertensive medications, in the right arm with an oscillometric monitor device $\left(\mathrm{OMRON}^{\circledR} \mathrm{H}-003 \mathrm{D}\right)$, with cuff adjusted for arm circumference. The mean of the last two measurements was used to estimate systolic and diastolic blood pressure.

Blood samples were drawn after a 12-h overnight fast for analysis of lipids (triglycerides, total, LDL and HDL cholesterol), high-sensitive C-reactive protein, and glycosylated hemoglobin (HbA1c). Triglyceride and HDL cholesterol measurements in participants who were receiving drug treatment for elevated plasma triglycerides levels and/or for reduced HDL cholesterol levels were not included in the analysis. At 8 am following a 10-12 h overnight fast, subjects received a 75-g oral glucose tolerance test with plasma glucose and insulin determinations at $0,30,60,90$ and $120 \mathrm{~min}$. In the 9 subjects who were taking oral hypoglycemic agents, the oral glucose tolerance test was performed 4 days after withdrawal of hypoglycemic medications. Fasting glucose was defined by the glucose concentration at $0 \mathrm{~min}$. All participants were classified according to glucose tolerance status [13] and presence of metabolic syndrome [5]. The methodology and procedures were reviewed to equate to STARD criteria, and the diagram to report flow of participants through the study is displayed in Additional file 2 [14].

\section{Classification of metabolic syndrome}

Metabolic syndrome was defined as the presence of 3 of 5 following: waist circumference $\geq 80 \mathrm{~cm}$ for women and $\geq 94 \mathrm{~cm}$ for men; serum triglyceride $\geq 1.7 \mathrm{mmol} / \mathrm{L}$ ) or receiving treatment for elevated serum triglycerides; $\mathrm{HDL}$ cholesterol $<1.0 \mathrm{mmol} / \mathrm{L}$ for men and $<1.3 \mathrm{mmol} / \mathrm{L}$ for women or receiving treatment for reduced HDL cholesterol; systolic blood pressure $\geq 130 \mathrm{mmHg}$ or diastolic blood pressure $\geq 85 \mathrm{mmHg}$ or receiving antihypertensive treatment; and fasting plasma glucose $\geq 6.1 \mathrm{mmol} / \mathrm{L}$ or receiving treatment for hyperglycemia, according to the Joint Interim Statement for the harmonization of metabolic syndrome criteria [5]. In addition, the American Diabetes Association criteria for impaired glucose tolerance ( $2 \mathrm{~h}$-plasma glucose $\geq 7.8 \mathrm{mmol} / \mathrm{L}$ ) was used as a criteria for hyperglycemia [13]. 


\section{Classification of glucose tolerance}

Based on HbA1c and fasting and $2 \mathrm{~h}$-plasma glucose concentrations, participants were categorized according to American Diabetes Association criteria as having normal glucose tolerance (fasting plasma glucose $<6.1 \mathrm{mmol} / \mathrm{L}$, $2 \mathrm{~h}$-plasma glucose level $<7.8 \mathrm{mmol} / \mathrm{L}$, and $\mathrm{HbA} 1 \mathrm{c}<5.7 \%$ [39 $\mathrm{mmol} / \mathrm{mol}]$ ), impaired fasting glucose (fasting plasma glucose $6.1-6.9 \mathrm{mmol} / \mathrm{L}$ and $2 \mathrm{~h}$-plasma glucose level $<7.8 \mathrm{mmol} / \mathrm{L}$ ); impaired glucose tolerance (fasting plasma glucose $<6.1 \mathrm{mmol} / \mathrm{L}$ and $2 \mathrm{~h}$-plasma glucose level 7.8-11.0 $\mathrm{mmol} / \mathrm{L}$ ), and diabetes (fasting plasma glucose $\geq 7.0 \mathrm{mmol} / \mathrm{L}$ and/or 2 -h $\mathrm{PG} \geq 11.1 \mathrm{mmol} / \mathrm{L}$ or HbA $1 \mathrm{c} \geq 6.5 \%$ [ $48 \mathrm{mmol} / \mathrm{mol}]$ or receiving medication for diabetes control) [13]. Participants with impaired fasting glucose and/or impaired glucose tolerance and/ or HbA1c between $5.7 \%$ (39 $\mathrm{mmol} / \mathrm{mol}$ ) and $6.4 \%$ $(48 \mathrm{mmol} / \mathrm{mol}$ ) were considered to have prediabetes.

\section{Estimation of insulin resistance indices}

Eleven static indices were analyzed: Bennet, fasting insulin $\left(\operatorname{Ins}_{0 \text { min }}\right)$, fasting insulin/fasting glucose ratio, fasting insulin resistance index (FIRI), fasting insulin sensitivity index $\left(\mathrm{ISI}_{0 \mathrm{~min}}\right)$, homeostasis model assessment (HOMA)IR, HOMA-2-IR, HOMA-2-IS, McAuley, quantitative insulin sensitivity check index (QUICKI) and Raynaud. Eight dynamic indices were analyzed: Avignon, Gutt, Matsuda, oral glucose insulin sensitivity index (OGIS), Stumvoll with and without demographics, $2 \mathrm{~h}$-insulin sensitivity index $\left(\mathrm{ISI}_{120 \mathrm{~min}}\right)$, and $2 \mathrm{~h}$-insulin/2 h-glucose ratio.

All indices were calculated according to published equations, as described in Additional file 1. The reciprocal of an IS index was used as equivalent to an insulin resistance index (1/insulin sensitivity index $=$ insulin resistance index). Two additional markers, adiponectin and HOMA-AD, were also examined because of their potential physiopathological association with metabolic syndrome [8]. Indices designed specifically to estimate beta-cell function were not considered.

\section{Statistical analysis}

Data were expressed as absolute number and \%, mean \pm standard deviation or median (P25-P75). Sample size analysis was performed considering an expected sensitivity of 0.73 and an expected specificity of 0.70 to identify metabolic syndrome, based on a study that evaluated the performance of the HOMA index [15], and on an expected prevalence of metabolic syndrome of 0.30 [6]. It was estimated that 182 subjects would be necessary to achieve a precision of 0.118 for sensitivity and 0.080 for specificity. Calculations were made by using an Excel spreadsheet available online [16]. To compare demographic, clinical, and laboratory data, using the presence of metabolic syndrome in our sample, the Chi square test and independent-sample $t$-test were used as appropriate. Variables with a non-normal distribution were $\log$ transformed before analysis. The accuracy of insulin resistance indices to identify the metabolic syndrome was determined by analyzing the area under the curve (AUC) in a receiver operating characteristic (ROC) curve. The AUC comparison for different insulin resistance indices was examined by the method proposed by DeLong et al. [17]. For each index, we determined an optimal cut point at the ROC curve based on the Youden index and distance to coordinate $(0,1)$. The Youden index is a common summary measure of the ROC curve. It was calculated as (Sensitivity + Specificity) -1 [18]. From this ideal cut point, we established the performance of these indices as a diagnostic test for the metabolic syndrome, including sensitivity, specificity, and positive and negative likelihood ratios. For practical clinical purposes, we estimated the probability of the outcome employing the Bayes nomogram. The pretest probability was defined as the prevalence of metabolic syndrome in our sample [19]. To deal with multiple testing, we performed a Bonferroni correction test [20]. The conventional $P$ value of 0.05 was divided by the total number of analyzed indices in the present study ( 21 indices), giving a $P$-value of 0.0024 for statistical significance. For one-to-one comparisons, i.e., in Table 1, it was considered $P<0.05$. Calculations were made by using SPSS (version 19.0; SPSS Inc., Chicago) and pROC package for R i386 (version 3.1.2.; R Foundation, Vienna).

\section{Results}

\section{Participants' characteristics}

The patient population was subdivided by the absence (23.5\%) or presence $(76.5 \%)$ of metabolic syndrome. Participants with metabolic syndrome were older and had lower adiponectin plasma levels, and higher HbA1c and high-sensitive $\mathrm{C}$-reactive protein levels. The groups did not differ by gender distribution, ethnicity, smoking habits, and physical activity status. As expected, the prevalence of prediabetes and type 2 diabetes was higher in the group with metabolic syndrome (Table 1).

\section{Accuracy of insulin resistance indices in the diagnosis of metabolic syndrome}

ROC analysis showed that the reciprocal of Gutt and OGIS indices yielded an AUC above 0.8, whereas most other indices gave an AUC between 0.7 and 0.8 (Fig. 1). However, there was no statistical difference between the reciprocal of Gutt AUC and the following 11 indices of AUC (OGIS, Matsuda, HOMA-AD, Avignon, ISI ${ }_{0 \min }$, HOMA-IR, FIRI, Bennet, HOMA-2-IS, fasting insulin, 
Table 1 Participants' demographic, clinical, and laboratory characteristics according to the presence of metabolic syndrome

\begin{tabular}{|c|c|c|c|}
\hline & \multicolumn{3}{|c|}{ Metabolic syndrome } \\
\hline & Absent & Present & $P$ value $^{a}$ \\
\hline Number (\%) & $43(23.5)$ & $140(76.5)$ & - \\
\hline Women $n(\%)$ & $35(81.4)$ & $99(70.7)$ & 0.235 \\
\hline Age (years) & $47.0 \pm 12.8$ & $54.1 \pm 11.1$ & $0.001^{d}$ \\
\hline White ethnicity $n(\%)^{b}$ & $34(79.1)$ & $110(78.5)$ & 0.811 \\
\hline Smoking $n(\%)$ & $5(11.6)$ & $17(12.1)$ & 0.617 \\
\hline Physical activity ${ }^{c}$ & & & 0.057 \\
\hline Sedentary n (\%) & $16(37.2)$ & $78(55.7)$ & - \\
\hline Light exercise $n(\%)$ & $18(41.9)$ & $42(30.0)$ & - \\
\hline Moderate exercise $n(\%)$ & $6(14.0)$ & $18(12.9)$ & - \\
\hline Heavy exercise $n(\%)$ & $3(7.0)$ & $2(1.4)$ & - \\
\hline $\mathrm{BMI}\left(\mathrm{kg} / \mathrm{m}^{2}\right)$ & $27.8 \pm 5.1$ & $32.3 \pm 5.9$ & $<0.05^{d}$ \\
\hline Overweight $n(\%)$ & $17(39.5 \%)$ & $50(35.7 \%)$ & $<0.05^{\mathrm{d}}$ \\
\hline Obesity n (\%) & $14(32.6 \%)$ & $83(59.2 \%)$ & $<0.05^{d}$ \\
\hline Waist circumference (cm) & & & $<0.05^{d}$ \\
\hline Women & $95.0 \pm 13.4$ & $103.0 \pm 12.2$ & \\
\hline Men & $93.8 \pm 11.4$ & $107.0 \pm 12.2$ & \\
\hline Glucose tolerance status & & & $<0.001^{d}$ \\
\hline Normal glucose tolerance $n(\%)$ & $39(90.7)$ & $19(13.6)$ & \\
\hline Prediabetes $n(\%)$ & $3(7.0)$ & $78(55.7)$ & \\
\hline Type 2 diabetes $n(\%)$ & $1(2.3)$ & $43(30.7)$ & \\
\hline Fasting plasma glucose $(\mathrm{mmol} / \mathrm{l})$ & $5.0(4.5-5.5)$ & $5.8(5.2-6.3)$ & $<0.05^{d}$ \\
\hline 2-h plasma glucose (mmol/l) & $6.1(4.9-6.9)$ & $9.5(8.3-11.6)$ & $<0.05^{d}$ \\
\hline $\mathrm{HbA1c}(\%)$ & $5.5(5.3-5.8)$ & $6.2(5.7-6.7)$ & $<0.001^{d}$ \\
\hline $\mathrm{HbA} 1 \mathrm{c}(\mathrm{mmol} / \mathrm{mol})$ & $37(34-40)$ & $44(39-50)$ & $<0.001^{d}$ \\
\hline HDL cholesterol (mmol/l) & $1.35(1.21-1.61)$ & $1.19(1.01-1.37)$ & $<0.05^{d}$ \\
\hline Triglycerides (mmol/l) & $1.11(0.78-1.52)$ & $1.45(1.11-1.99)$ & $<0.05^{\mathrm{d}}$ \\
\hline High-sensitive C-reactive protein (nmol/l) & $15.2(5.7-31.4)$ & $38.1(12.4-80.0)$ & $<0.001^{d}$ \\
\hline Adiponectin $(\mu \mathrm{g} / \mathrm{mL})$ & $16.5(10.4-21.8)$ & $11.0(7.9-14.0)$ & $0.001^{d}$ \\
\hline Systolic blood pressure $(\mathrm{mm} \mathrm{Hg})$ & $123.5(115-135)$ & $142.5(128.3-161.1)$ & $<0.05^{d}$ \\
\hline Diastolic blood pressure $(\mathrm{mm} \mathrm{Hg})$ & $78.7 \pm 11.2$ & $87.6 \pm 13.1$ & $<0.05^{d}$ \\
\hline \multicolumn{4}{|l|}{ Medications } \\
\hline Antihypertensive $n(\%)$ & $9(20.9)$ & $75(53.5)$ & $<0.001^{d}$ \\
\hline Statin $n(\%)$ & $4(9.5)$ & $24(17.1)$ & 0.325 \\
\hline Hypoglycemic $n(\%)$ & $0(0)$ & $9(6.4)$ & 0.164 \\
\hline \multicolumn{4}{|l|}{ Insulin resistance indices } \\
\hline 2 h-insulin/2 h-glucose ratio & $0.27(0.40-0.70)$ & $0.60(0.37-0.96)$ & 0.141 \\
\hline Fasting insulin $\left(I \mathrm{~ns}_{0 \mathrm{~min}}\right)$ & $7.7(4.9-10.3)$ & $12.5(7.9-18.3)$ & $<0.001^{b}$ \\
\hline Fasting insulin/fasting glucose ratio & $0.08(0.05-0.10)$ & $0.12(0.07-0.17)$ & $0.013^{b}$ \\
\hline FIRI & $1.5(1.0-2.2)$ & $3.0(1.8-4.3)$ & $<0.001^{b}$ \\
\hline HOMA-IR & $1.6(1.1-2.4)$ & $3.3(2.0-4.8)$ & $<0.001^{b}$ \\
\hline HOMA-2-IR & $0.1(0.1-0.2)$ & $0.25(0.20-0.40)$ & $<0.001^{b}$ \\
\hline Matsuda & $2.2(1.6-3.2)$ & $3.6(2.8-5.1)$ & $<0.001^{\mathrm{b}}$ \\
\hline \multicolumn{4}{|l|}{ Insulin sensitivity indices } \\
\hline Avignon & $14.0(9.2-26.2)$ & $6.2(4.1-9.6)$ & $<0.001^{b}$ \\
\hline Bennet & $0.6(0.5-0.7)$ & $0.45(0.39-0.55)$ & $<0.001^{b}$ \\
\hline Gutt & $4.5(3.7-5.4)$ & $2.4(2.1-3.2)$ & $<0.001^{b}$ \\
\hline HOMA-2-IS & $692(525-1081)$ & $410(278-654)$ & $<0.001^{b}$ \\
\hline
\end{tabular}


Table 1 (continued)

\begin{tabular}{|c|c|c|c|}
\hline & \multicolumn{3}{|c|}{ Metabolic syndrome } \\
\hline & Absent & Present & $P$ value ${ }^{a}$ \\
\hline$|S|_{0 \min }$ & $15.1(10.3-22.8)$ & $7.5(5.2-11.8)$ & $<0.001^{\mathrm{b}}$ \\
\hline$|S|_{120 \mathrm{~min}}$ & $69.8(27.7-40.4)$ & $60.9(37.2-95.9)$ & 0.141 \\
\hline McAuley & $8.3(7.5-9.7)$ & $7.1(6.3-8.3)$ & $0.001^{b}$ \\
\hline OGIS & $422(379-467)$ & $325(276-371)$ & $<0.001^{\mathrm{b}}$ \\
\hline QUICKI & $3.1(3.0-3.4)$ & $2.9(2.8-3.1)$ & 0.059 \\
\hline Raynaud & $5.2(3.9-8.2)$ & $3.2(2.2-5.0)$ & $<0.001^{\mathrm{b}}$ \\
\hline Stumvoll with demographics & $12.2(9.6-15.2)$ & $14.1(2.2-27.8)$ & $<0.001^{b}$ \\
\hline Stumvoll without demographics & $0.5(0.5-1.0)$ & $0.3(0.25-0.50)$ & $<0.001^{b}$ \\
\hline \multicolumn{4}{|l|}{ Other indices } \\
\hline Adiponectin & $16.5(10.0-22.9)$ & $11.1(8.2-14)$ & $0.001^{b}$ \\
\hline HOMA-AD & $2.4(1.6-3.9)$ & $6.1(3.5-12.4)$ & $<0.001^{b}$ \\
\hline
\end{tabular}

Data are expressed as the absolute number, \% or mean \pm standard deviation or median (P25-75)

a $\mathrm{P}$ value for comparisons between two groups was tested by $\mathrm{X}^{2}$ test for categorical variables or Student's $t$-test for continuous variables

b Ethnicity was recorded as white or non-white, which included black $(n=13)$, brown $(n=9)$, yellow $(n=0)$, indigenous $(n=3)$ and undeclared $(n=14)$

c Participants reported the frequency of exercise in four categories, adapted from the classification proposed by Tuomilehto et al. [12]

d Significant statistical difference $(P<0.05)$

and Raynaud; $P \geq 0.0024$ ), indicating that these equations provide an index of insulin resistance that identifies individuals with the metabolic syndrome (Fig. 2; Additional file 3).

Since subjects with metabolic syndrome were older than subjects without the condition, we stratified our sample based on median of age and performed an additional analysis. For subjects younger than 53 years old, there was no statistical difference between the reciprocal of Gutt AUC and 16 indices of AUC; while for subjects older than 53 years old, there was no difference for 17 indices (Additional file 4).

Additionally, we performed a stratified analysis of AUC values for subjects with and without obesity and with normal and large waist circumference. Generally, Gutt still presented the largest AUC, but more indices were statistically equivalent to Gutt. When the subgroup of patients with obesity is analyzed, Gutt and OGIS present a great performance (AUC 0.930 and 0.924).

\section{Performance of insulin resistance indices as diagnostic tests for metabolic syndrome}

Using the ideal cut point, we determined the sensitivity and specificity of each equation in demonstrating the presence of metabolic syndrome. The reciprocals of Gutt and OGIS, which had the greatest AUC values in predicting the metabolic syndrome, and HOMA-IR and fasting insulin, which are commonly employed indices for insulin resistance, are displayed in Fig. 2 and in
Additional file 3. Using an optimal cut point of 0.268 for the reciprocal of Gutt, this index gave sensitivity of $86.4 \%$ (80.1-91.4\%; CI 95\%) and specificity of $76.7 \%$ (68.2-87.6\%) to identify the metabolic syndrome. For a positive test result (test value above the cut point), the post test probability of identifying metabolic syndrome was 92 , which means a $20 \%$ increase in the probability of identifying metabolic syndrome when Gutt has a positive result. For a negative test result (test value below the cut point), the post test probability was $37 \%$, which means a $51 \%$ decrease in the probability of identifying metabolic syndrome when Gutt has a negative result. In comparison, for a positive HOMA-IR test result, the post test probability of identifying metabolic syndrome was $90 \%$ (increase of $17 \%$ ); for a negative test result, the post test probability was 53\% (decrease of $30 \%)$. The same procedure was applied to other indices (Fig. 2; Additional file 3).

\section{Discussion}

In the present study, we evaluated the ability of all published indices of insulin resistance to identify the metabolic syndrome. From the quantitative standpoint, the Gutt index had the greatest area under the ROC curve (Fig. 1). However, 11 other indices, most notably the dynamic ones, were statistically non-inferior to the Gutt index. The inability to demonstrate statistically significant differences between those 12 indices of insulin resistance most likely is related to the conservative method utilized to define significance. Because 21 differences were 


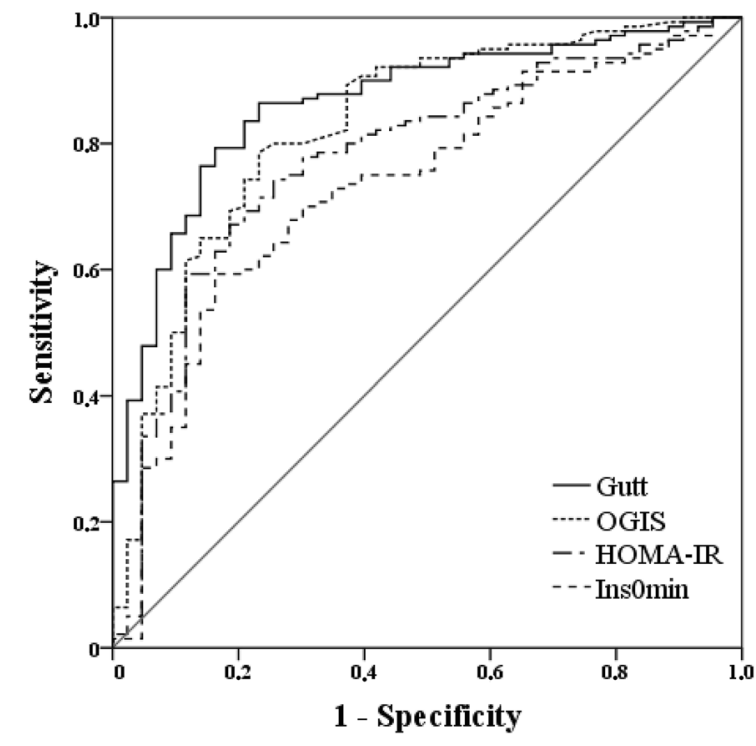

Fig. 1 ROC curves of insulin resistance indices used to identify the metabolic syndrome. The two indices with greater area under the curve (AUC) in our analysis (Gutt and OGIS) and the two most frequently used indices in clinical practice and other research studies (HOMA-IR and fasting plasma insulin concentration) are displayed

evaluated, the alpha $(\mathrm{P}<0.05)$ was split by 21 , requiring a $\mathrm{P}<0.0024$ to demonstrate significance. If one-to-one comparisons are made using $\mathrm{P}<0.05$, the Gutt index outperforms all other indices, except OGIS. OGIS had a similar performance when the sample was stratified by age, BMI and waist circumference (Additional file 4). It should be noted that the Gutt index, although validated against the euglycemic insulin clamp, primarily included subjects who were obese and had normal glucose tolerance. Hanley et al. [21] found that the Gutt index demonstrated the best overall ability to predict type 2 diabetes compared to 18 other indices in a large multiethnic cohort. A Finnish study showed that Gutt was not statistically inferior to other indices when tested against the gold standard measure of insulin sensitivity obtained by the hyperinsulinemic euglycemic clamp [22].

Additionally, we found that adiponectin, an insulin sensibilizing hormone, and high-sensitive C-reactive protein, a marker of subclinical inflammation, were different in subjects with and without metabolic syndrome. The insulin sensitizer and anti-inflammatory role of adiponectin is a result of several actions: suppression of hepatic gluconeogenesis, activation of AMPK and fatty acid oxidation in skeletal muscle, suppression of the inflammatory response through the enhancement of nitric oxide synthase activity in endothelial cells and inhibition of the expression of adhesion molecules, such as VCAM-1, E-selectin, and ICAM [23]. In a previous study, we found that adiponectin levels decreased with increasing number of metabolic syndrome criteria, and it is in part determined by its relationship with abdominal adiposity [24]. Additionally, adiponectin levels were inversely related to HDL levels and high-sensitive $\mathrm{C}$-reactive protein and positively related to blood pressure levels [25]. These findings were confirmed in an additional analysis within this study, where the number of metabolic syndrome criteria were greater in subjects more insulin resistant than those more insulin sensitive categorized by the median of insulin resistance indices (Additional file 5). From a physiopathological perspective, inflammation inhibits insulin action through the release of cytokines and adipocytokines and contributes to the progression from insulin resistance to the development of hyperglycemia [26], which explain the higher rates of dysglycemia and diabetes in the group with metabolic syndrome. These findings also corroborate the role of adipokines and inflammation in the pathogenesis of metabolic syndrome [25].

The present study has some limitations. First, our sample was composed by a predominantly White female population with a high prevalence of metabolic syndrome. It would be important to confirm these findings in further studies, in populations with a more diverse ethnic background, more balanced gender distribution, and a lower prevalence of metabolic syndrome. Second, methods for insulin measurement are not standardized [8]. Although we used a reliable assay with appropriate controls, these results might not be applicable to other studies, which use different assays to measure plasma insulin. Third, we did not perform euglycemic insulin clamps in the present study. It would have been of great interest to see how well the insulin clamp predicts the metabolic syndrome in comparison to the other 21 insulin resistance indices that were evaluated.

In summary, a variety of indices provide a measure of insulin resistance that can be used for clinical and epidemiological research studies. Because the equations used to estimate insulin resistance rely on different variables, i.e., hyperglycemia, hyperinsulinemia, and, in some cases, adiposity and demographic parameters, they reflect different components of the underlying physiopathological disturbances present in the metabolic syndrome. Thus, it is not surprising that there may be variability in their ability to predict the metabolic syndrome. 

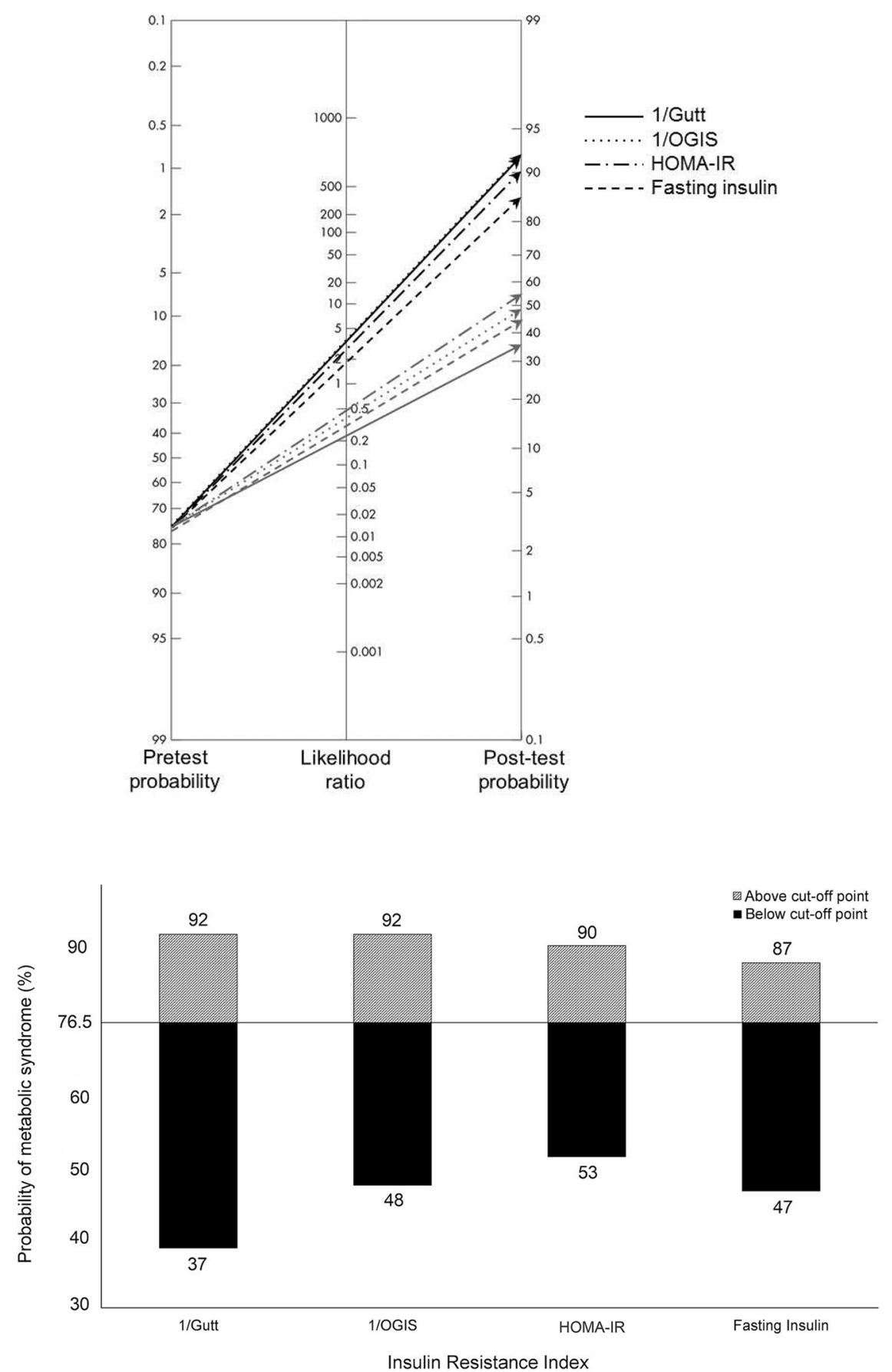

Fig. 2 Fagan's likelihood nomograms show the variation in probability of a metabolic syndrome diagnosis after a positive (above cut-off point) or negative test (below cut-off point) result for the reciprocals of Gutt and OGIS indices, as well as HOMA-IR and fasting insulin indices. The figure below synthesize these findings, comparing the variation of probability for metabolic syndrome between indices [Nomograms were adapted from Fagan TJ (N Engl J Med 1975;293:257; copyright 1975, New England Journal of Medicine, all rights reserved)] 


\section{Additional files}

Additional file 1. Development and validation method of the equations for insulin resistance indices. This file contains a table that describes the development and validation method of each insulin resistance index. It also contains comments regarding their performance and applications in previous studies.

Additional file 2. Flow diagram. This diagram shows the flow of participants through the study.

Additional file 3. ROC curve analysis of insulin resistance indices used to identify metabolic syndrome/Performance of selected insulin resistance indices as diagnostic tests for metabolic syndrome (Cl 95\%). The first table shows the AUC values of insulin resistance indices to identify metabolic syndrome and their statistical comparison to the reciprocal of Gutt AUC, which had the best AUC. The second table shows the sensitivity, specificity, likelihood ratios and positive and negative predictive values of selected equations in identifying presence of metabolic syndrome.

Additional file 4. Performance of insulin resistance indices to identify metabolic syndrome in a stratified analysis by age, BMI and waist circumference. The tables show the performance of insulin resistance indices in subgroups stratified by age, BMI and waist circumference.

Additional file 5. Number of metabolic syndrome criteria by median of insulin sensitivity and resistance indices. The tables show a comparison between groups divided by median of selected insulin sensitivity and resistance indices for number of metabolic syndrome criteria.

\section{Abbreviations}

AUC: area under the curve; FIRl: fasting insulin resistance index; HbA1c: glycosylated hemoglobin; HOMA: homeostasis model assessment; Ins ${ }_{0 \text { min }}$ : fasting insulin; $\mid \mathrm{SI}_{\mathrm{Omin}}$ : fasting insulin sensitivity index; $\mathrm{ISI}_{120 \mathrm{~min}}$ : 2 h-insulin sensitivity index; OGIS: oral glucose insulin sensitivity index; QUICKI: quantitative insulin sensitivity check index; ROC: receiver operating characteristic.

\section{Authors' contributions}

LPA reviewed the literature relating to the various indices of insulin resistance and wrote the manuscript. BLN, TCP and LdAM coassisted in researching the data and reviewed the manuscript. Fernando Gerchman discussed the results with all others, and edited the manuscript. All authors read and approved the final manuscript.

\section{Author details}

1 Scientific Initiation Program, Faculty of Medicine, Federal University of Rio Grande do Sul, Rua Ramiro Barcelos, 2400, Second floor, Porto Alegre, RS 90035-003, Brazil. ${ }^{2}$ Metabolism Unit, Endocrinology Division, Hospital de Clínicas de Porto Alegre, Rua Ramiro Barcelos, 2350, Building 12, Fourth floor, Porto Alegre, RS 90035-003, Brazil.

\section{Acknowledgements}

Prof. Ralph Anthony DeFronzo for his critical review and helpful feedback of this study. The manuscript was edited to conform to scientific English by the English Language Editing service from Elsevier's Web Shop.

\section{Competing interests}

The authors declare that they have no competing interests.

\section{Availability of data and materials}

The datasets used and/or analyzed during the current study are available from the corresponding author on reasonable request.

\section{Consent for publication}

Not applicable.

\section{Ethics approval and consent to participate}

All procedures performed in studies involving human participants were in accordance with the ethical standards of the institutional and/or national research committee and with the 1964 Helsinki declaration and its later amendments or comparable ethical standards. Informed consent was obtained from all individual participants included in the study.

\section{Funding}

This work was supported by the Rio Grande do Sul Research Foundation (FAPERGS; Grant Number 5989.284.18921.12062013), the Coordination of Higher Education and Graduate Training (CAPES; Grant Number 486802/20132), the Brazilian National Research Council (CNPq; Number 486802/2013-2), and the Hospital de Clínicas de Porto Alegre Research and Events Incentive Funds (FIPE-HCPA). Luciana P. Antoniolli received a scientific initiation scholarship from the Rio Grande do Sul Research Foundation and the Brazilian National Research Council.

\section{Publisher's Note}

Springer Nature remains neutral with regard to jurisdictional claims in published maps and institutional affiliations.

Received: 18 January 2018 Accepted: 11 August 2018

Published online: 20 August 2018

\section{References}

1. Berson S, Yalow R. Insulin agonists and insulin resistance. In: Ellenberg $M$, Rifkin $\mathrm{H}$, editors. Diabetes mellitus: theory and practice. New York: McGraw-Hill; 1970. p. 388-423.

2. Kahn SE, Hull RL, Utzschneider KM. Mechanisms linking obesity to insulin resistance and type 2 diabetes. Nature. 2006. https://doi.org/10.1038/ nature05482.

3. Ford ES. Risks for all-cause mortality, cardiovascular disease, and diabetes associated with the metabolic syndrome: a summary of the evidence. Diabetes Care. 2005;28(7):1769-78.

4. Malik S, Wong N, Franklin S, Kamath T, L'Italien G, Pio J, et al. Impact of the metabolic syndrome on mortality from coronary heart disease, cardiovascular disease, and all causes in United States adults. Circulation. 2004. https://doi.org/10.1161/01.CIR.0000140677.20606.0E.

5. Alberti K, Eckel RH, Grundy SM, Zimmet PZ, Cleeman Jl, Donato KA, et al. Harmonizing the metabolic syndrome: a joint interim statement of the International Diabetes Federation Task Force on Epidemiology and Prevention; National Heart, Lung, and Blood Institute; American Heart Association; World Heart Federation; International Atherosclerosis Society; and International Association for the Study of Obesity. Circulation. 2009. https ://doi.org/10.1161/CIRCULATIONAHA.109.192644.

6. Vidigal F, Bressan J, Babio N, Salas-Salvadó J. Prevalence of metabolic syndrome in Brazilian adults: a systematic review. BMC Public Health. 2013. https://doi.org/10.1186/1471-2458-13-1198.

7. Defronzo RA. Glucose intolerance and aging: evidence for tissue insensitivity to insulin. Diabetes. 1979;28(12):1095-101.

8. Singh B. Surrogate markers of insulin resistance: a review. World J Diabetes. 2010;1(2):36.

9. Liu R, Christoffel KK, Brickman WJ, Liu X, Gadgil M, Wang G, et al. Do static and dynamic insulin resistance indices perform similarly in predicting pre-diabetes and type 2 diabetes? Diabetes Res Clin Pract. 2014. https:// doi.org/10.1016/j.diabres.2014.04.014.

10. Stumvoll M, Mitrakou A, Pimenta W, Jenssen T, Yki-Jarvinen H, Haeften TV, et al. Use of the oral glucose tolerance test to assess insulin release and insulin sensitivity. Diabetes Care. 2000:23(3):295-301.

11. Petruccelli J, Saboia A. Caracteristícas étnico-raciais da populacão: classificacões e identidades - número 2. Rio de Janeiro: Instituto Brasileiro de Geografia e Estatística; 2013.

12. Tuomilehto J, Lindström J, Eriksson JG, Valle TT, Hämäläinen H, IlanneParikka P, et al. Prevention of type 2 diabetes mellitus by changes in lifestyle among subjects with impaired glucose tolerance. N Engl J Med. 2001. https://doi.org/10.1056/NEJM200105033441801.

13. American Diabetes Association. Executive summary: standards of medical care in diabetes-2009. Diabetes Care. 2008. https://doi.org/10.2337/ dc09-s013.

14. Bossuyt PM, Reitsma JB, Bruns DE, Gatsonis CA, Glasziou PP, Irwig L, et al. STARD 2015: an updated list of essential items for reporting diagnostic accuracy studies. BMJ. 2015. https://doi.org/10.1136/bmj.h5527. 
15. Gayoso-Diz P, Otero-González A, Rodriguez-Alvarez MX, Gude F, García F, Francisco AD, et al. Insulin resistance (HOMA-IR) cut-off values and the metabolic syndrome in a general adult population: effect of gender and age: EPIRCE cross-sectional study. BMC Endocr Disord. 2013. https://doi. org/10.1186/1472-6823-13-47.

16. Naing L. Sample size calculation for sensitivity \& specificity studies. In: Universiti Sains Malaysia. 2004. http://www.kck.usm.my/ppsg/statistica I_resources/samplesize_forsensitivity_specificitystudies_inNaing.xls. Accessed 07 Mar 2018.

17. Delong ER, Delong DM, Clarke-Pearson DL. Comparing the areas under two or more correlated receiver operating characteristic curves: a nonparametric approach. Biometrics. 1988;44(3):837.

18. Ruopp MD, Perkins NJ, Whitcomb BW, Schisterman EF. Youden index and optimal cut-point estimated from observations affected by a lower limit of detection. Biom J. 2008. https://doi.org/10.1002/bimj.200710415.

19. Kramer CK, Zinman B, Gross JL, Canani LH, Rodrigues TC, Azevedo MJ, et al. Coronary artery calcium score prediction of all cause mortality and cardiovascular events in people with type 2 diabetes: systematic review and meta-analysis. BMJ. 2013. https://doi.org/10.1136/bmj.f1654.

20. Armstrong RA. When to use the Bonferroni correction. Ophthalmic Physiol Opt. 2014. https://doi.org/10.1111/opo.12131.

21. Hanley AJ, Williams K, Gonzalez C, D'agostino RB, Wagenknecht LE, Stern MP, et al. Prediction of type 2 diabetes using simple measures of insulin resistance: combined results from the San Antonio Heart Study, the Mexico City Diabetes Study, and the Insulin Resistance Atherosclerosis Study. Diabetes. 2003;52(2):463-9.

22. Lorenzo C, Haffner SM, Stančáková A, Laakso M. Relation of direct and surrogate measures of insulin resistance to cardiovascular risk factors in nondiabetic finnish offspring of type 2 diabetic individuals. J Clin Endocrinol Metab. 2010. https://doi.org/10.1210/jc.2010-1144.

23. Ruscica M, Baragetti A, Catapano AL, Norata GD. Translating the biology of adipokines in atherosclerosis and cardiovascular diseases: gaps and open questions. Nutr Metab Cardiovasc Dis. 2017. https://doi. org/10.1016/j.numecd.2016.12.005.

24. Frankenberg ADV, Nascimento FV, Gatelli LE, Nedel BL, Garcia SP, Oliveira $\mathrm{CV}$, et al. Major components of metabolic syndrome and adiponectin levels: a cross-sectional study. Diabetol Metab Syndr. 2014. https://doi. org/10.1186/1758-5996-6-26.

25. Frankenberg ADV, Reis AF, Gerchman F. Relationships between adiponectin levels, the metabolic syndrome, and type 2 diabetes: a literature review. Arch Endocrinol Metab. 2017. https://doi.org/10.1590/2359-39970 00000316.

26. Shulman Gl. Ectopic fat in insulin resistance, dyslipidemia, and cardiometabolic disease. N Engl J Med. 2014. https://doi.org/10.1056/NEJMr a1011035.
Ready to submit your research? Choose BMC and benefit from:

- fast, convenient online submission

- thorough peer review by experienced researchers in your field

- rapid publication on acceptance

- support for research data, including large and complex data types

- gold Open Access which fosters wider collaboration and increased citations

- maximum visibility for your research: over $100 \mathrm{M}$ website views per year

At $\mathrm{BMC}$, research is always in progress.

Learn more biomedcentral.com/submissions 